\title{
Posibles incrementos del uso de energías renovables en el desarrollo de fábricas de azúcar como biorrefinerías
}

Erenio González Suárez ${ }^{1}$, Ana C. de Armas Martinez², Marlen Morales Zamora ${ }^{3}$, Mayra Guzmán Villavicencio ${ }^{4}$

\section{Resumen:}

En el trabajo se explica cómo mediante el cambio de esquemas tecnológicos y de usos de las materias primas y corrientes intermedias se puede incrementar el uso de fuentes renovables de energía en la transformación paulatina de fábricas de azúcar a biorrefinería. Finalmente se presentan conclusiones para el trabajo futuro.

Palabras claves: energías renovables, biorrefinería, fábricas de azúcar.

\section{Abstract:}

In the work it is explained how by means of the change of technological outlines and of uses of the matters cousins and intermediate currents you can increase the use of renewable sources of energy in the gradual transformation of factories of sugar to biorefinery. Finally conclusions are presented for the future work.
Keywords: renewable energy, biorefinery, factories of sugar.

\section{Introducción}

Es necesario reducir la dependencia de los recursos petroquimicos, mediante el uso de la biomasa como fuente de energías y materias primas renovables. La industria de la caña de azúcar presenta una posibilidad de desarrollo de este sector agroindustrial y se impone como necesidad y posibilidad de conversión de fábricas de azúcar en biorrefinerías con la gestión del conocimiento en especial la intensificación paulatina de las energías renovables. Aquí es necesario considerar que por cada tonelada de caña de azúcar cortada llegan a la fábrica de azúcar son: $104 \mathrm{~kg}$ de azúcar, $430 \mathrm{Kg}$ de agua, $231 \mathrm{~kg}$ de bagazo, $33 \mathrm{~kg}$ de cachaza y $26 \mathrm{~kg}$ de miel (Gálvez, 1988), por lo que la principal fuente de energía renovables para

\footnotetext{
1) Universidad Central "Marta Abreu" de Las Villas, Santa Clara, Villa Clara, Cuba Email: erenio@uclv.edu.cu 2) Universidad Central "Marta Abreu” de Las Villas, Santa Clara, Villa Clara, Cuba. Email:anaceliam@uclv.edu.cu 3) Universidad Central "Marta Abreu" de Las Villas, Santa Clara, Villa Clara, Cuba Email: marlenms@uclv.edu.cu 4) Empresa Ronera Central, Villa Clara, Santo Domingo, Cuba Email:innovación@cubaron.co.cu
} 
el desarrollo de una biorrefinería está en el uso adecuado de las diferentes fracciones de la caña de azúcar. El paso inicial en la intensificación de un proceso es mejorar el aprovechamiento de los recursos disponibles y la estrategia para ello es el objetivo de este trabajo.

\section{Materiales y Métodos}

Metodológicamente este trabajo descasa en una estrategia fundamentadas en el análisis y síntesis de los procesos de la Industria Química evaluando alternativas de operación basada en datos extraídos de procesos industriales, en el concepto de que no solo se debe, sino también, se puede investigar en la industria. Se aplican diseños experimentales para evaluar los efectos de cambios en las tecnologías actuales

\section{Resultados}

En los procesos tecnológicos establecidos de producción de azúcar, sin duda uno de los primeros pasos para su desarrollo como biorrefinería es la instalacion aleda- ña de una destilería de etanol, producto que aconsejamos líder en el desarrollo de una biorrefinería debido a que tiene valor de uso en sí mismo pero también como materia prima en la llamada Ruta Alcoquímica y para la producción de bebidas, siendo factibles en cada instalación industrial evaluar acciones asociadas al proceso de producción de azúcar y derivados, con una visión de integración material y energética de proceso, a saber:

\section{1) Impacto del Jugo de los filtros en los balances materiales y energéticos}

En las tecnologías más establecidas de fabricación de azúcar crudo se recicla el 100 $\%$ de los jugos filtrados después de la clarificación lo que permite mayor obtención de azúcar, sin embargo, las alternativas de extraer diferentes por cientos de jugo de los filtros del proceso y no reciclarlosdan lugar a varias opciones de operación y uso de mezclas de mieles y jugo de los filtros en la producción de etanol mediante la mezcla de sustratos según se resume en la Tabla 1

Tabla 1. Por cientos de producción según capacidad instalada (PCI) y de consumo de vapor según reciclado de jugo de los filtros (JF).

\begin{tabular}{|c|c|c|c|c|}
\hline $\begin{array}{c}\text { \% de reciclado } \\
\text { del JF }\end{array}$ & $\begin{array}{c}\text { \% de PCI de } \\
\text { Azúcar base } 96\end{array}$ & $\begin{array}{c}\% \text { de Miel total } \\
\text { producida }\end{array}$ & $\begin{array}{l}\text { \% de uso } \\
\text { de vapor. }\end{array}$ & $\begin{array}{l}\% \text { Bagazo } \\
\text { sobrante }\end{array}$ \\
\hline 100 & 100.00 & 100 & 100 & 100.00 \\
\hline 80 & 97.74 & 97.75 & 97.76 & 121.96 \\
\hline 40 & 93.20 & 93.22 & 92.78 & 171.09 \\
\hline 0 & 88.62 & 88.63 & 87.79 & 220.00 \\
\hline
\end{tabular}


Esto permite tener un crecimiento en el bagazo para electricidad y vapor de la producción de azúcar con relativos bajos descensos en la producción de azúcar y miel

2) Mezcla de jugos y mieles como sustratos en la producción de bioetanol

La diminución de mieles, así como la disponibilidad de Jugo de los Filtros invita a la solución tecnológica de em- plear mezclas de ambos para la producción de etanol en la producción de etanol. Aplicando una matriz experimental de Enrejado Simplex (Sheffe; 1958), para mezclas de Jugo de los filtros (JF), Jugo Secundario (JS) y miel (M) según el mezclado de sustratos se considero su efecto en el Rendimiento y la Eficiencia en la fermentación como se muestra en la Tabla 2

Tabla 2. Resultados de cada muestra.ART Inicial: $120(\mathrm{~g} / \mathrm{L})$

\begin{tabular}{|c|c|c|c|}
\hline Corrida & ART Final(g/L) & Rendimiento\% & Eficiencia\% \\
\hline JS & 11,49 & 45,89 & 71,265 \\
\hline JS+ M & 4,34 & 45,22 & 70,215 \\
\hline JF+JS & 4,33 & 43,75 & 67,927 \\
\hline JF + M & 7,50 & 50,58 & 78,537 \\
\hline J F & 8,79 & 46,13 & 71,629 \\
\hline $\mathbf{M}$ & 9,59 & 46,19 & 71,726 \\
\hline $\mathbf{M + J S + J F}$ & 6,017 & 50,88 & 79,014 \\
\hline
\end{tabular}

Aquí se obtiene desde el punto de vista energético y económico el ahorro de agua en la dilución de mieles, que se logra con los jugos, lo que brinda un valor estratégico para el desarrollo de una biorrefinería utilizando recursos renovables.

\section{Producción de etanol de segunda generación}

Como se comprende, el incremento del bagazo sobrante, asociado a la diminución de mieles induce a utilizar el bagazo como materia prima para la producción de etanol.

La producción de etanol de segunda generación utilizando bagazo como materia prima ha permitido lograr un licor azucarado susceptible de ser mezclado con mieles y otras corrientes secundarias, lo que se hace más viable con el sobrante de bagazo obtenido de la extracción del jugo de los filtros. Para 
el estudio de mezclas de sustratos azucarados de primera y segunda generación se realiza un diseño de mezclas enrejado simplex, con la variable respuesta\% alcohólico en volumen (ALC, Y1).

La tabla 3 muestra los resultados, donde para todos los puntos se obtienen resultados de por cientos alcohólicos, en el orden de los obtenidos con mieles.

E1 modelo que describe la influencia en el parámetro de respuesta $\% \mathrm{Alc}(\mathrm{Y})$ de las variables de mezclas de los diferentes sustratos y permite maximizar su valor $Y$ que responde a la mezcla de los tres componentes, con la siguiente composición: X1:0,299, X2:0,342 y X3:0,358, para un \% alcohólico de 5,26 (Morales et al, 2018).
Los residuos lignocelulósicos obtenidos de la hidrólisis enzimática, enriquecidos en lignina pueden ser destinado a otras producciones (Morales et al; 2016) o ser quemados con un alto valor calórico en beneficio de la sustentación energética de la biorrefinería, aquí es de interés conocer que el poder calórico disponible de los desechos de la producción de etanol a partir de 1 tonelada de bagazo es determinado según la ecuación propuesta por Demirbas (2004) es de 8751.43 MJ, mientras que inicialmente esa tonelada tenía un poder calórico de $17835 \mathrm{MJ}$, por lo tanto la energía potencial disponible es el $49.07 \%$ de la que se tendría quemando todo el bagazo directamente, lo que es una contribución al balance energético de la biorrefinería.

Tabla3.Resultados del diseño de mezclas de sustratos azucarados de primera y segunda generación aplicando un diseño Enrejado Simplex.

\begin{tabular}{|c|c|c|c|c|}
\hline Puntos & MIEL X1 & JF X2 & HIDMX3 & \%ALCY1 \\
\hline 1 & 1,00 & 0,00 & 0,00 & 5,38 \\
\hline 2 & 0,00 & 1,00 & 0,00 & 4,39 \\
\hline 3 & 0,00 & 0,00 & 1,00 & 4,31 \\
\hline 4 & 0,50 & 0,50 & 0,00 & 4,68 \\
\hline 5 & 0,50 & 0,00 & 0,50 & 4,78 \\
\hline 6 & 0,00 & 0,50 & 0,50 & 5,06 \\
\hline 7 & 0,33 & 0,33 & 0,33 & 5,29 \\
& & & &
\end{tabular}




\section{4) Producción de biogás de las vina- zas de destilería para generación de va- por.}

Aquí una alternativa a evaluar es la obtención de biogás mediante la digestión anaeróbica del residual integrado de la destilería con un potencial energético de.

Los productos de valor agregado que se producen en una planta de biogás son: a) Biogás con valor energético de 722 $\mathrm{Kca} / \mathrm{H} 1$ de etanol producido (Guzmán et al, 2017), b) Lodo microbiológico de valor fertilizante, c) Efluente tratado que puede utilizarse como agua de riego.

\section{5) Producción de biodiesel de residuos sólidos (Cacbaza)}

La producción de cachaza ha sido utilizada como abono y también como fuente de productos químicos, pero previamente pueden ser separados sus diferentes fracciones y con ellas lograr la obtención productos químicos de interés y biodiesel. Se ha logrados un rendimiento de 0,0786 1 de biodiesel $/ \mathrm{kg}$ de cachaza que es factible de ser empleado

en las operaciones agrícolas o de transportación de la industria de la caña de azúcar con un impacto económico positivo. (García, 2012).

\section{Discusión}

Los resultados aquí mostrados permiten revelar las potencialidades de uso de la biomasa cañera para sustento del desarrollo paulatino de la industria de la caña de azúcar como biorrefinería, resalta el hecho de que una decisión tecnológica pueda duplicar la disponibilidad de bagazo en una instalacion industrial, permitiendo abrir el diapasón de este material como materia prima para etanol, fuente de la ruta alcoquímica o para combustible logrando un sustento energético de las producciones con un también agradable aseguramiento de agua para las nuevas producciones. La producción de biogás de las vinazas de destilería es fuente de energía renovable para el sustento de una biorrefinería.

La obtención de productos químicos y biodiesel de la cachaza residual, así como el biogás de las vinazas refuerza el concepto de biorrefinería y su sustento energético a partir del fraccionamiento adecuado de la caña de azúcar de manera sustentable.

E1 uso de energía solar puede ser un complemento para las demandas de electricidad en cada instalacion (Guzmán et al; 2018)

\section{Conclusiones}

Es factible el incremento paulatino del uso de las energías renovables provenientes de la propia biomasa cañera en las biorrefinerías.

Con el fraccionamiento del bagazo se logran producciones, en paralelo con la de etanol, a partir de los azucares de 5 átomos de carbono y permite disponer de una masa

lignocelulolítica, que permite contribuir a garantizar la electricidad y el vapor requerido.

Es necesario optimizar a futuro en cada instalacion la integración de los procesos. 


\section{Referencias bibliográficas}

Demirbas A; A. H. Demirbas. (2004) Estimating the calorific values of Lignocelulosic Fuels. Energy exploration \& exploitation. Volume 22, Number 2. 2004. 135- 143

1) García Prado, R. (2012) "Estrategia para la producción de biocombustibles en Guatemala"Tesis en opción al grado científico de Doctor en Ciencias Técnicas. UCLV, Cuba,

2) Gálvez Taupier, L. (1988) Manual de los derivados de la caña de azúcar. GEPLACEA, México

3) Guzmán Villavicencio, M., E. González Suárez, M. Morales Zamora. (2017) Metodología para la gestión de la tecnología y la innovación y su integración con el análisis de procesos en la industria ronera cubana.Convención Científica Internacional Universidad Central "Marta Abreu" de Las Villas
4) Morales-Zamora, M, E. González Suárez, L. Mesa Garriga (2016) Avances en la obtención de tableros de fibras a partir de mezclas de residuales lignocelulósicos de bagazo. AFINIDAD Vol. 73, 575, julio setiembre.

5) Morales Zamora, M, A. C .de Armas Martinez, L. Mesa Garriga, D. Acosta Martínez, E. González Suárez. (2018). Avances en el uso del licor hidrolizado de bagazo en la fermentación de mezclas azucaradas AFINIDAD Vol. 75, 581, Enero- marzo

6) Scheffe, H. (1958) "Experiment with mixture". Journal of Royal Statistical Society". Vol.20.311-360.

\section{Agradecimientos:}

A la Red RibERA por su invitación. 